\title{
A Case of Ectopic Posterior Lobe of the Pituitary without Stalk Transection and a History of Breech Delivery
}

\author{
Tomohiro Someya, Tomoyuki Watanabe, Toshiyuki Yasuda and Hiroo Niimi \\ Department of Pediatrics, School of Medicine Chiba University, Chiba, Japan
}

Key words: pituitary stalk transection, ectopic posterior lobe, GH deficiency

\section{Introduction}

Pituitary gland organogenesis is a fusion between the adenohypophysis, which derives from the neuroectoderm of the rostral neural fold, and the neurohypophysis, which develops as a downward projection of the neuroectoderm of the base of the brain. Magnetic resonance (MR) imaging of the brain in idiopathic GH deficiency often reveals pituitary stalk transection and ectopic posterior lobe of the pituitary gland (1). The formation of ectopic posterior lobe of the pituitary gland is usually associated with traumatic transection of the pituitary stalk caused by breech delivery, perinatal asphyxia and brain trauma. The endocrinological studies of these patients suggest that there must be reestablishment of the hypothalamo-hypophyseal portal circulation (2), and regeneration of the nerve fibers of the hypothalamo-neurohypophyseal tract depends on the level at which the stalk was sectioned (2). Here we report a Japanese boy of GH deficiency with ectopic posterior lobe of the pituitary gland without stalk transection.

Correspondence: Dr. Tomohiro Someya, Department of Pediatrics, School of Medicine Chiba University, 1-8-1 Inohana, Chuo-ku, Chiba 260, Japan

\section{Case Report}

The patient is the first child of healthy unrelated Japanese parents of normal stature. He was born by caesarian section because of cephalopelvic disproportion after a 40 -week pregnancy. His birth weight and length were $3840 \mathrm{~g}$ and $52 \mathrm{~cm}$, respectively. His developmental milestones were normal and he had no history of hypoxia, hypoperfusion, asphyxia or brain trauma. He had never had symptomatic hypoglycemia. At 9 yr 4 mo of age, he was referred to us because of short stature and nocturnal enuresis. His height and bone age were $117.5 \mathrm{~cm}(-2.5 \mathrm{SD})$ and $6 \mathrm{yr}$. He showed no signs of puberty and his testicular volume was $1.5 \mathrm{~mL}$ each. The growth curve is shown in Fig. 1. Thyroid and adrenal functions were normal (Table 1). The plasma IGF-1 concentration was $0.37 \mathrm{U} / \mathrm{mL}$. The $\mathrm{GH}$ concentration in the first voided urine was $90.0 \mathrm{pg} / \mathrm{mg}$. Cr. Thyroid function, evaluated by basal T4 and T3 concentrations and the TSH level in response to TRH infusion, was normal. Gonadotropin levels in response to LH-RH infusion were within the normal pre-pubertal range. He had poor GH response to insulin-induced hypoglycemia (peak serum GH concentration $2.6 \mu \mathrm{g} / \mathrm{L}$ ) and arginine infusion (8.3 $\mu \mathrm{g} / \mathrm{L})$. In view of these results, GH treatment was started with recombinant human GH (0.5 IU/ $\mathrm{kg} /$ week). An overnight water deprivation test 
Table 1 Endocrinological loading studies

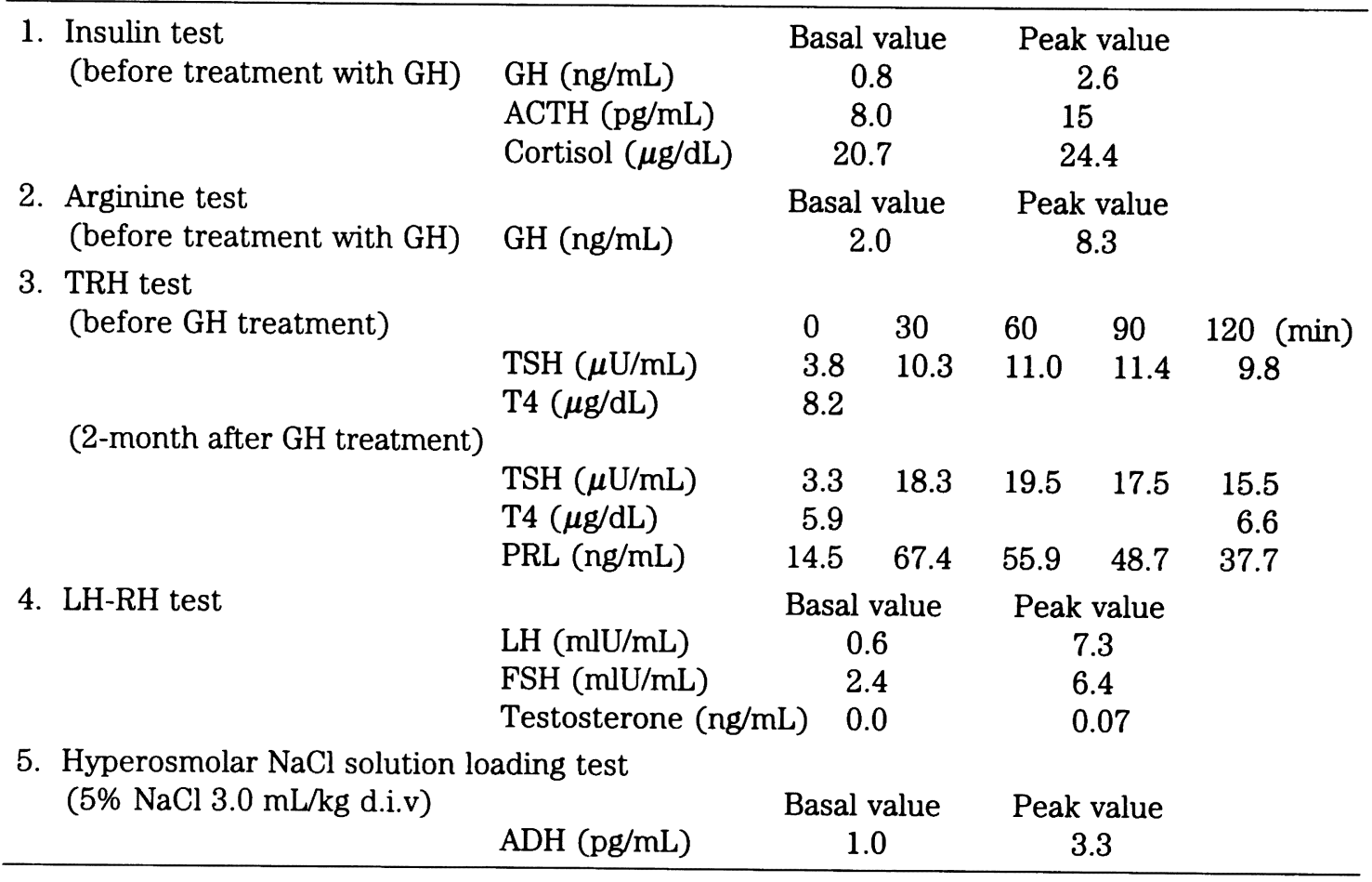

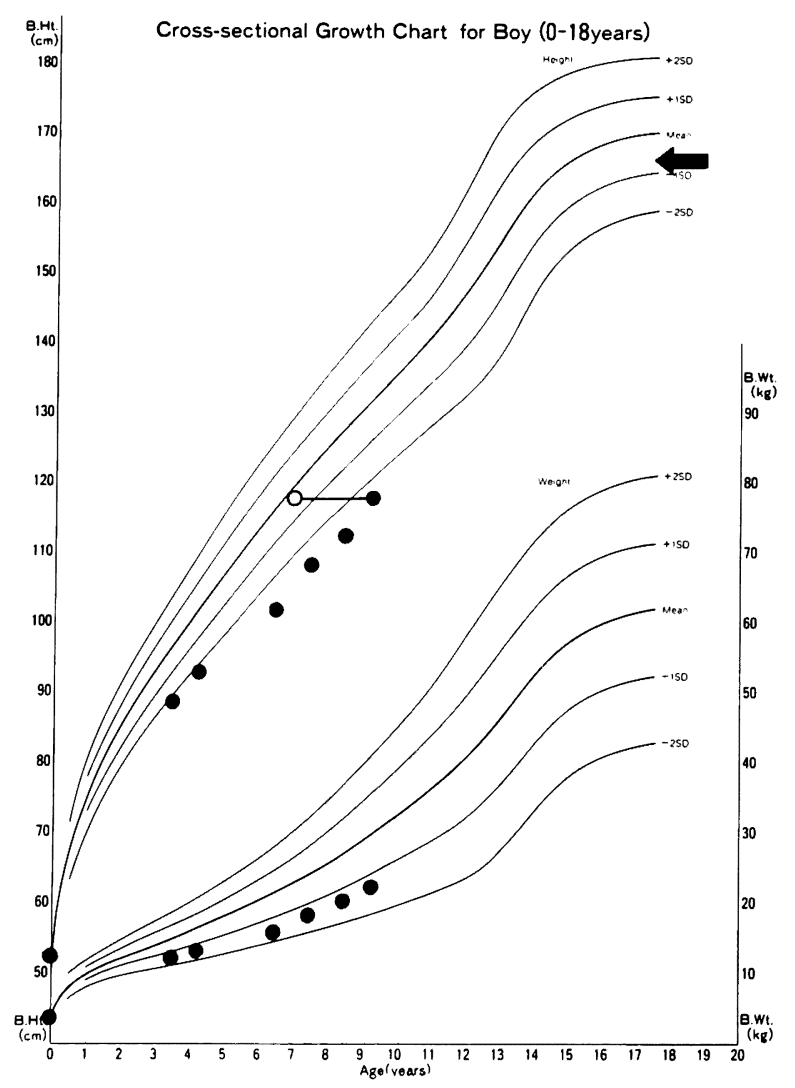

showed a urinary specific gravity of 1021. The maximal plasma $\mathrm{ADH}$ level in response to the infusion of hyperosmolar $\mathrm{NaCl}$ solution was $3.3 \mathrm{pg} /$ $\mathrm{mL}$. Nocturnal enuresis was relieved by intranasal DDAVP administration. After treatment with $\mathrm{GH}$, the TSH level at $120 \mathrm{~min}$ after TRH infusion was $15.5 \mu \mathrm{U} / \mathrm{mL}$. Magnetic resonance imaging revealed the formation of an ectopic posterior lobe, but not with pituitary stalk transection. The pituitary height and length were $1.0 \mathrm{~mm}$ (control 2.8-4.0 mm) and $2.1 \mathrm{~mm}$ (control 5.0-7.5 mm), respectively (Fig. 2).

\section{Discussion}

The patient's condition is summarized as small anterior pituitary gland, normal stalk size,

Fig. 1 Growth curve of the patient. The closed circles, open circle and arrow indicate chronological age, bone age and target height, respectively. 


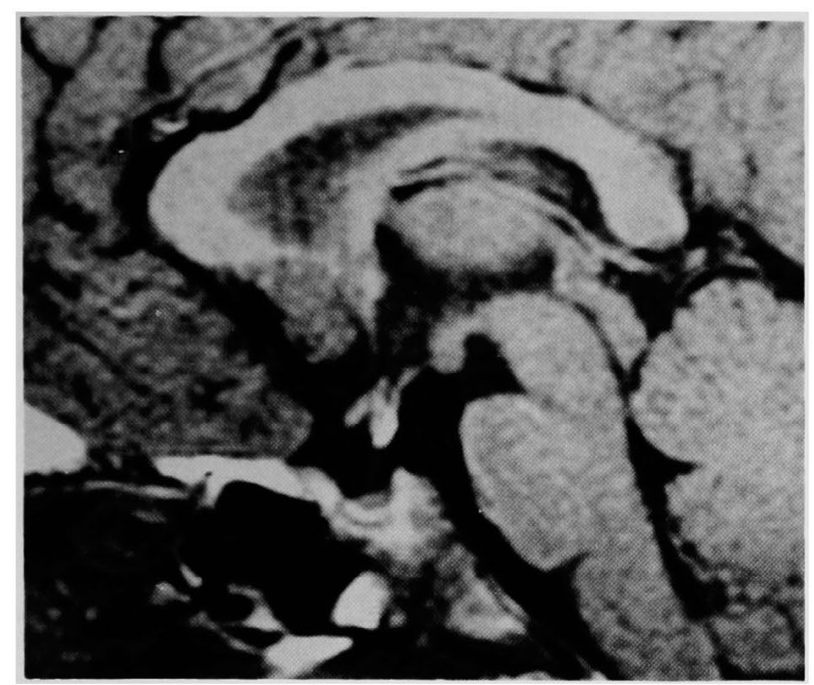

Fig. 2 Sagittal T1-weighted magnetic resonance image of the brain of this patient. The ectopic posterior lobe and the pituitary stalk are shown.

ectopia of the posterior lobe, GH deficiency and a sustained high TSH concentration after a TRH infusion test. ADH, ACTH, LH and FSH levels were normal.

The pathogenesis of ectopic posterior lobe was in general explained by traumatic transection of the pituitary stalk during delivery (2), but a recent study conducted in $101 \mathrm{GH}$ defi- cient patients revealed posterior pituitary ectopia (PPE) in 59 patients, and PPE patients showed a higher male/female ratio (3.2), a greater frequency of multiple pituitary hormone deficiency and congenital abnormalities, but only $32 \%$ of PPE patients were born by breech delivery (3). Thus this case indicates that a defective induction of the mediobasal structure of the brain in the early embryo could account for both the complex morphological MRI abnormality and clinio-endocrinological features in some patients with ectopic posterior pituitary gland.

\section{References}

1. Fujisawa I, Kikuchi K, Nishimura K, Togashi K, Itoh K, Noma S, et al. Transection of the pituitary stalk: development of an ectopic posterior lobe assessed with MR imaging. Radiology 1987; 165: 487-9.

2. Kikuchi K, Fujisawa I, Momoi T, Yamanaka C, Kaji M, Nakano Y, Konishi J, et al. Hypothalamic-pituitary function in growth hormone-deficient patients with pituitary stalk transection. J Clin Endocrinol Metab 1988; 67: 817-23.

3. Triulizi F, Scotti G, di Natale B, Pellini C, Lukezic $\mathrm{M}$, Scognamiglio $\mathrm{M}$, et al. Evidence of a congenital midline brain anomaly in pituitary dwarfs: a magnetic resonance imaging study in 101 patients. Pediatrics 1994; 93:409-16. 